All letters are subject to editing and may be shortened. Letters should be sent to the BJGP office by e-mail in the first instance, addressed to

journal@rcgp.org.uk (please include your postal address). Alternatively, they may be sent by post as an MS Word or plain text version on CD or DVD. We regret that we cannot notify authors regarding publication. Letters not published in the Journal may be posted online on our Discussion Forum. For instructions please visit: http://www.rcgp.org.uk/bjgp-discuss

\section{Bell's palsy and Lyme disease}

I read with interest Richard Davenport et al's useful and informative editorial on Bell's palsy in the August BJGP.' It may be useful for GPs to be aware of some differences in the aetiology and therefore management of children with Bell's palsy for us on the south coast. As many will be aware, the New Forest in Hampshire near Southampton has traditionally been a hot spot for Lyme disease. Lyme disease can present in many ways; neuroborreliosis is the most common secondary manifestation of Lyme, and Bell's palsy the commonest presentation of neuroborreliosis. It is clear, however, that the tick which causes Lyme's is now more widespread and over the last 2 years in Winchester, north of the New Forest, we have seen cases of Lyme meningoencephalitis, arthritis, and multiple cases of Bell's palsy. A significant proportion of Bell's palsy have positive Lyme's serology; so much so that some of our consultants are considering Amoxil ${ }^{\circledR}$ or doxycycline (in the over 12's) as early 'blind' treatment options as well as the more traditional prednisolone with or without aciclovir as described in the editorial. Of note is the fact that very few of the group of Lyme serology positive Bell's palsy have had a history of a tick bite although many live in rural areas. Tick bites in children are often in the hair, hence very difficult to find and correctly identify.

I thought this information might be of use to GPs in the south, and perhaps with global warming may be more useful further afield!

\section{Simon Struthers,}

Paediatric Consultant, Paediatrics
Department, Mailpoint 07, Royal Hampshire County Hospital, Romsey Road, Winchester, SO22 5DG.

E-mail: Simon.Struthers@wehct.nhs.uk

\section{REFERENCE}

1. Davenport RJ, McKinstry B, Morrison JM, et al. Bell's palsy; new evidence provides a definitive drug therapy strategy. Br J Gen Pract 2009; 59: 569-570.

DOI: 10.3399/bjgp09X472692

\section{Early experience}

Ten months ago our practice commenced an 'extended hours' service intended to serve patients unable to attend the surgery during working hours. Some colleagues chose to work an evening surgery while I elected to start early. I can remember back in October 2008 the first patient, an 80-year-old retired gentleman, arriving yawning and bleary eyed for a review of his regular medication. Upon asking how he was he replied, 'Terrible doctor, do I really need to come this early to see you? They said it was the only slot left!'.

Now almost 1 year into the extended hours we decided to review its usage. We looked back over the last 6 months of a once-weekly 07.40 Tuesday surgery start.

Between 1 March 2009 and 31 August 2009, 34 pre-8 a.m. appointments were booked. There were four DNAs, 14 appointments for new problems, and 16 for follow-up and ongoing patient management. The mean age of patients was 44 years with no patient older than 66 years and the youngest aged 16 years. Five patients had taken up early appointments on more than one occasion. The majority of appointments were for women, $24 / 30(80 \%)$.

The ratio of women to men is in keeping with the rest of my morning surgery appointments for the same 6month period 638/846 (75\%). Although the numbers involved are small, DNAs for the early morning appointments was $12 \%(4 / 34)$, twice the rest of the morning surgery, $5.6 \%$ (50/896).

Although the DNA rate will need to be reviewed in the future, it certainly seems that early starts suit particular patients well, as is indicated by repeated booking of these slots by some of them. It is apparent that patients of a working age are those taking up these appointments and the reception staff must be thanked for their role in helping the adaptations to the new surgery times. As the dark winter mornings draw in, we will see if these trends continue.

\section{Geryl Rees,}

Heaton Mersey Medical Practice, 460 Didsbury Road, Stockport, Cheshire, SK4 3BT.

\section{John Whitaker,}

Heaton Mersey Medical Practice, Stockport. E-mail: johnwhitaker@doctors.net.uk

\section{REFERENCE}

1. Department of Health. Final guidance: extended hours access scheme directed enhanced service.

http://www.dh.gov.uk/en/Publicationsandstatistics/Pu blications/PublicationsPolicyAndGuidance/DH_0875 57 (accessed 14 Sep 2009).

DOI: 10.3399/bjgp09X472700

\section{ENT 2-Week Wait audit}

Interested in the effectiveness of the 2Week Wait (2WW) referral system, we 